\title{
PREDICTORS OF OBSTACLE COURSE RACING PERFORMANCE
}

original paper

(c) University School of Physical Education in Wroclaw

DOI: https://doi.org/10.5114/hm.2020.89914

\author{
WESTON TITUS ${ }^{1}$, RICHARD F. ARMENTA ${ }^{2}$, MATTHEW M. SCHUBERT ${ }^{2}$ \\ ${ }^{1}$ Department of Health, Kinesiology, and Recreation Management, Palomar College, San Marcos, USA \\ ${ }^{2}$ Department of Kinesiology, California State University, San Marcos, USA
}

\section{ABSTRACT}

Purpose. Obstacle course racing (OCR) has become a popular recreational activity in the recent 10 years, with more than 8.5 million participants. Despite the popularity, little is known about predictors of performance in OCR; research to date has focused on injury prevalence. The purpose of this study was to conduct laboratory and field tests of athletic performance in OCR athletes and examine their relationships to performance in simulated OCR.

Methods. Overall, 32 men and women (mean \pm standard deviation age: $42 \pm 10$ years; OCR experience: $2.8 \pm 2.3$ years) completed laboratory testing for maximal oxygen uptake, anaerobic power (Wingate), vertical jump, flexibility, and body composition. Additional field tests were performed for 400-meter and 1-mile running time, muscle strength (back squats and deadlifts), and endurance (bucket carry for distance), grip strength, and burpees. The participants also completed a 3-mile simulated OCR. Independent t-tests examined differences between sex, and bivariate regressions were conducted between testing variables and OCR performance.

Results. For the combined sample, the best individual predictors were mean relative power from the Wingate tests $(\beta \pm$ standard error $[S E]:-6.47 \pm 1.12)$ and mile-run time $(\beta \pm S E$ : $6.43 \pm 0.71)$. Multivariable analysis controlling for age, sex, and mile-run time found an independent association between bucket carry for distance and race time $(\beta \pm S E:-0.04 \pm 0.01)$, but mile-run time was still the best predictor $(\beta \pm S E: 6.33 \pm 0.97)$.

Conclusions. Data from the study suggest that aerobic and anaerobic fitness have important contributions to OCR success. Key words: obstacle course racing, performance prediction, running, functional training

\section{Introduction}

Obstacle courses (OCs) have been used for training, competition, and fitness promotion since antiquity. This is likely due to the fact that OCs require individuals to exhibit high levels of all aspects of fitness cardiovascular endurance, muscular strength and endurance, agility, and flexibility [1, 2]. Additional components can include coordination, balance, and problem-solving ability [2]. OCs have been used as military training tools and in physical education programs, but recent years have shown an increase in OCs emerging as popular competitions for the general public - now known as obstacle course racing (OCR) [1]. For example, Spartan has, since 2007, had more than 5 million participants complete races; Tough Mudder, a competing OCR company, reports upwards of 3.5 million participants to date [3-5]. OCRs come in many types, but Spartan has standardized several of them. Sprint races are $5 \mathrm{~km}$ and include 20-25 obstacles [3]. Super races are $10 \mathrm{~km}$ and include 25-30 obstacles [3]. Beast races are approximately half-marathon length with 30-35 obstacles [3]. Ultras are $50 \mathrm{~km}$ with ca. 60 obstacles [3]. Tough Mudder includes $5 \mathrm{~K}$ races and their 'classic' race, which is 8-10 miles [4]. Obstacles comprise elements like rope climbs, hurdles, monkey bars, mud pits, sandbag or bucket carries for distance, spear throwing, cargo nets, climbing walls, and other obstacles [3-5]. The terrain also is generally very rugged, including substantial elevation changes, and the running surface can involve a combination of dirt, loose sand, mud, and grass [3-5]. Finally, in 'elite' and 'age-

Correspondence address: Matthew M. Schubert, Department of Kinesiology, California State University,

333 S. Twin Oaks Valley Road, San Marcos, CA, USA, e-mail: mschubert@csusm.edu

Received: September 4, 2019

Accepted for publication: October 22, 2019

Citation: Titus W, Armenta RF, Schubert MM. Predictors of obstacle course racing performance. Hum Mov. 2020;21(2):51-57; doi: https://doi.org/10.5114/hm.2020.89914. 
group' race categories, athletes are penalized with 30 burpees if they are unable to complete an obstacle. All of these factors present considerable challenges for participants in order to successfully complete OCRs, particularly because athletes must have a strong aerobic background and adequate muscle strength and endurance.

OCR as a sport combines aerobic endurance training with bouts of high-intensity functional training (HIFT) in the form of the obstacles that athletes encounter. Research on predictors of OCR performance is lacking. However, studies have been conducted on predictors of long-distance running and HIFT workouts. For instance, in trained runners, maximal oxygen uptake $\left(\mathrm{VO}_{2} \max \right)$ explained $81 \%$ of the variance in 10-mile race time, while the velocity at $\mathrm{VO}_{2}$ max was the best predictor of race time. Conversely, in HIFT workouts, aerobic fitness $\left(\mathrm{VO}_{2} \max \right)$, maximal rate of anaerobic ATP production (peak Wingate power), and muscle strength (1-repetition max for back squat, deadlift, shoulder press) have been associated with HIFT workouts lasting ca. 3-15+ minutes [6-8].

Although most research examining OCR has been limited to injury studies, a recent case report sheds some light on the physiological demands of the sport [9]. In the case study, the participant spent ca. $59 \%$ of a 24-hour OCR competition in moderate intensity exercise based on accelerometry, equal to an average intensity of 3.4 METs [9]. In addition, the individual expended ca. 244 calories per hour, which indicates a significant energetic challenge over the entire 24-hour competition [9]. This suggests a heavy reliance on aerobic energy metabolism during OCR. Though the authors reported interrupted heart rate data, the participant reached a peak heart rate of ca. 95\% maximum [9]. Thus, even though accelerometry did not detect vigorous activity, it is highly probable that there were sections where the participant was utilizing high rates of anaerobic metabolism as well.

Given the increased popularity in OCR, it is worthwhile for athletes and coaches to know what the best physiological predictor(s) of OCR performance would be in order to optimize training. Therefore, the purpose of this study was to conduct a series of laboratory and gym-based tests in experienced OCR athletes and examine their associations with simulated OCR performance. We hypothesized that aerobic fitness $\left(\mathrm{VO}_{2} \max \right)$ would be the strongest predictor of simulated OCR performance. Additionally, we hypothesized that muscle strength and endurance and anaerobic power would also be related to OCR performance.

\section{Material and methods}

\section{Participants}

Healthy men and women $(n=13$ and 19, respectively) aged 18-60 years were recruited from a local gym focused on HIFT and OCR. Inclusion criteria involved a minimum 3-month history of OCR participation, no handicaps or physical disabilities preventing participation in moderate-vigorous physical activity, no history of cardiometabolic diseases, not currently being on medication influencing blood pressure or metabolism, and participating in at least 2 training sessions per week. All participants completed the informed consent form, as well as the Physical Activity Readiness Questionnaire (PAR-Q) and health history questionnaire before performing any testing.

\section{Experimental design}

This was an observational, cross-sectional study. Data were collected from September to December 2018. The participants completed an online survey, a laboratory assessment, gym-based testing, and an OCR simulation at the gym. The study and all tests were designed by the investigators in consultation with the gym owner and coaches.

\section{Questionnaire}

The online questionnaire was administered via a secure website by using Qualtrics software (Qualtrics XM, Qualtrics, Provo, USA). The participants answered demographic questions, health history questions, and questions about OCR experience.

\section{Laboratory testing}

The subjects reported to the Functional Performance Laboratory once where a battery of tests were conducted. They were instructed to arrive at least 3 hours post-prandial and to consume $350-500 \mathrm{ml}$ of water before their visit to ensure they were hydrated. The testing began with height obtained from a stadiometer, followed by weight and body composition determined with a multi-frequency bioelectrical impedance device (InBody 770, InBody USA, Cerritos, USA). Then, a flexibility test (sit and reach) was conducted followed by a vertical jump test. For the sit and reach test, the American College of Sports Medicine procedures were applied [10]; the results of 3 trials were recorded and averaged. For the vertical jump test, the 
participants performed a standing vertical jump. They were instructed to drop into the bottom of a squat position and explode vertically while hitting vanes on a vertical jump measuring device (Vertec); the results of 3 trials were recorded and averaged. The subjects then had a heart rate transmitter (Garmin HRM+, Garmin, Inc., Overland Park, USA) placed on their chest and were allowed to warm up on a treadmill for 5 minutes at a self-selected pace. At the end of the warm-up, they were fitted with a facemask and turbine connected to a metabolic cart (Cosmed Quark RMR/CPET, Cosmed Inc., Rome, Italy). They then began exercising at a comfortable running/jogging pace and $0 \%$ gradient. Every 2 minutes, the gradient was increased by $2 \%$ until volitional exhaustion. Breath by breath gas exchange data were recorded and averaged at 15-second intervals, and $\mathrm{VO}_{2}$ max was determined by averaging $\mathrm{VO}_{2}$ values of the final minute of exercise; secondary criteria included a respiratory exchange ratio $\geq 1.10$, heart rate $\pm 10 \mathrm{bpm}$ of age-predicted maximum (220 - age), and rate of perceived exertion $\geq 9$. The participants were given a 3-minute cool-down at a slow walk, then 10 minutes of rest. After the rest period, they mounted a cycle ergometer (Velotron Dynafit Pro, RacerMate Inc., Seattle, USA) for a Wingate test. They completed 30 seconds of 'all out' cycling at 7.5\% of their body weight, and peak power, mean power, and fatigue index were recorded.

\section{Gym testing}

For gym testing, the participants were asked to follow similar procedures to those they completed before lab testing. Gym tests were selected on the basis of conversations with experienced OCR trainers and racers who maintained that the tests accurately reflected the demands of OCR racing (i.e. endurance, grip strength, etc.). Gym testing consisted of $4 \times 400$-meter runs with 1-minute rests between intervals, a weighted bucket carry for maximal distance in 5 minutes ( 31.75 and $18.14 \mathrm{~kg}$ for men and women, respectively), a maximal hang test from a pull-up position while rotating grip every 5 seconds, maximum repetition back squats at $60 \%$ of body weight in 5 minutes, maximum repetition deadlifts at $120 \%$ of body weight in 5 minutes, and maximum repetition burpees in 5 minutes. Regarding the arm hang test, the participants alternated between pronated and supinated grip on the pull-up bar every 5 seconds until fatigue, which was signalled by the subject dropping from the bar. For the back squat, the athletes lifted the barbell from a squat rack, and then squatted below parallel $\left(90^{\circ}\right)$ before standing back up to full extension to be counted as a full repetition. For the deadlift, the participants grasped the barbell and lifted from the ground to a standing position. The weight was then returned to the ground in a controlled manner to be considered a successful repetition. The variables recorded were fastest 400-meter time in seconds, 1-mile-run time in minutes (sum of $4 \times 400$ minus the 1-minute rest periods), distance covered during the bucket carry in meters, hang time in seconds, and reps completed in 5 minutes for the final 3 movements. The subjects were allocated 5-minute recoveries between each test and were familiar and competent in all movements as determined by the investigators and gym coaches. The coaches led all participants through a standardized 15-minute warm-up including jogging, calisthenics, and stretching before testing.

\section{OCR simulation}

Finally, the participants returned to the gym on a separate day to complete a simulated OC race. The simulated race covered 5 kilometers and included 30 obstacles. The obstacles were similar or identical to those normally experienced during OCR, but the terrain was relatively flat, and the running surface largely asphalt and concrete instead of dirt, mud, or grass. A schematic representation of the race is shown in Figure 1. A detailed description of most obstacles is available from Spartan Race at https://www.spartan. com/en/spartan-race-obstacles. The course was constructed by OC design specialists certified through Spartan Race.

\section{Statistical analysis}

Descriptive statistics were calculated and included means and standard deviations. The results were computed for men and women combined and separately. Independent $t$-tests were run to determine differences in demographic, physiological, strength, and performance characteristics by sex. Effect sizes for sex differences are reported as Cohen's $d$, and may be interpreted as trivial $(<0.20)$, small $(0.20-0.39)$, moderate (0.40-0.79), and large ( $\geq 0.80)$ [11]. Bivariate linear regression was used to determine factors associated with race time completion. Additional linear regression models were run to adjust all associations for age, sex, and 1-mile-run time to determine factors independently associated with race time completion. Multicollinearity was assessed by using variance inflation factors with a cut-off $>4$ indicating potential collinearity be- 
W. Titus, R. Armenta, M. Schubert, Obstacle course racing performance

\begin{tabular}{|c|c|c|c|}
\hline \multicolumn{4}{|c|}{ 800-meter run } \\
\hline Mt. Olympus $\longleftrightarrow \longrightarrow$ & $2.4 / 1.8$ meter over wall $\rightarrow$ & Twister & Cargo hoist (45.35/31.75 kg) \\
\hline Z-wall & Z-wall & Z-wall & Z-wall \\
\hline Tarzan & Monkey bars & Rope climb (3 meters) & Rings +10 hand-release push-ups \\
\hline A-Frame & A-Frame & A-Frame & A-Frame \\
\hline Hurdles & Hurdles & Hurdles & Hurdles \\
\hline 100-meter bucket carry & 100-meter farmer carry & 100-meter pipe carry & $100-$ meter ball carry $(22.68 / 13.61 \mathrm{~kg})$ \\
\hline 10-meter broad jumps & 10-meter broad jumps & 10-meter-broad jumps & 10-meter broad jumps \\
\hline 800-meter run & 800-meter run & 800-meter run & 800-meter run \\
\hline \multicolumn{4}{|c|}{$\begin{array}{l}2 \text { tire flips }(158.73 / 113.38 \mathrm{~kg}) \\
10 \text { jumping ball slams }(9.07 / 6.8 \mathrm{~kg}) \\
\text { 200-meter run }\end{array}$} \\
\hline
\end{tabular}

Figure 1. Simulated obstacle course race

tween covariates. Where covariates were considered collinear, we made decisions to include only one in the adjusted model. All statistical analyses were conducted with the SAS 9.4 software (SAS Institute, Inc., Cary, USA).

\section{Ethical approval}

The research related to human use has complied with all the relevant national regulations and institutional policies, has followed the tenets of the Declaration of Helsinki, and has been approved by the California State University San Marcos Human Subjects Institutional Review Board (1295017-1).

\section{Informed consent}

Informed consent has been obtained from all individuals included in this study.

\section{Results}

A total of 32 participants completed all testing. They were $42 \pm 10$ years old, with $2.8 \pm 2.3$ years of experience with OCR. Overall subjects' characteristics, and characteristics stratified by sex, are shown in Table 1. In general, men were taller, heavier, and leaner. Men also performed better for the runs and had higher scores for $\mathrm{VO}_{2} \mathrm{max}$, vertical jump, and Wingate tests. Women performed better in the bucket carry and flexibility testing. Although the difference between men and women in simulated OCR performance equalled ca. 9 minutes, it was not statistically significant owing to a range of abilities in both sexes.

Simple bivariate regression revealed a number of variables linked to simulated OCR performance (Table 2). In the general sample, the best individual predic- tors were mean relative power from the Wingate tests and mile-run time. A $1 \mathrm{~W} / \mathrm{kg}$ increase in mean relative Wingate power was associated with a 6.47-minute reduction in race time, while a 1-minute increase in mile-run time was associated with a 6.43-minute rise in race time. Other significant predictors for the entire sample and stratified by sex are shown in Table 2 . When we controlled for age, sex, and 1-mile-run time, the only variable that was significantly associated with race time was the bucket carry (Table 3). A further distance covered in the bucket carry was associated with a 0.04 minute (ca. 2 seconds) faster race time. SGX training program performance trended towards a significant association with race time, with more reps completed being associated with a 0.2 minute (ca. 12 seconds) faster race time.

\section{Discussion}

OCR has grown explosively since companies such as Spartan Race (2007) and Tough Mudder (2010) were founded. Despite this, aside from injury surveillance data, little work has examined the characteristics of OCR participants and correlates of performance. To our knowledge, our study was the first to fill this knowledge gap by conducting lab and field tests of experienced OCR athletes. Our results revealed that traditional contributors of endurance performance success (i.e. aerobic fitness) are important to OCR success, but variables such as anaerobic power and muscle endurance are also essential.

The traditional model of endurance running performance includes $\mathrm{VO}_{2} \mathrm{max}$, running economy, fractional utilization of $\mathrm{VO}_{2} \max$, and the lactate threshold [12]. A study of 17 distance runners indicated that 
W. Titus, R. Armenta, M. Schubert, Obstacle course racing performance

Table 1. Demographic, physiological, strength, and performance characteristics of participants, stratified by sex $(n=32)$

\begin{tabular}{|c|c|c|c|c|c|}
\hline Variable & $\begin{array}{c}\text { Total } \\
\text { Mean }(S D) \\
n=32\end{array}$ & $\begin{array}{c}\text { Male } \\
\text { Mean }(S D) \\
n=13\end{array}$ & $\begin{array}{c}\text { Female } \\
\text { Mean }(S D) \\
n=19\end{array}$ & $\begin{array}{l}\text { Effect size } \\
\text { (Cohen's } d \text { ) }\end{array}$ & $p$ value \\
\hline Race time (min) & $44.53(10.59)$ & $39.25(8.09)$ & $48.14(10.74)$ & -0.91 & 0.36 \\
\hline Age (years) & $41.97(9.72)$ & $43.9(11.2)$ & $40.63(8.61)$ & 0.34 & 0.36 \\
\hline $\operatorname{BMI}\left(\mathrm{kg} / \mathrm{m}^{2}\right)$ & $24.21(2.47)$ & $24.83(2.85)$ & $23.79(2.15)$ & 0.42 & 0.25 \\
\hline Height (m) & $1.69(0.09)$ & $1.76(0.08)$ & $1.63(0.06)$ & 1.89 & $<0.001^{*}$ \\
\hline Body mass (kg) & $68.79(9.35)$ & $76.35(6.94)$ & $63.61(6.99)$ & 1.83 & $<0.001^{*}$ \\
\hline Fat mass $(\mathrm{kg})$ & $12.88(5.46)$ & $9.92(4.98)$ & $14.90(4.92)$ & -1.01 & $0.01^{*}$ \\
\hline Lean mass (kg) & $55.91(9.97)$ & $66.43(5.31)$ & $48.72(4.25)$ & 3.77 & $<0.001^{*}$ \\
\hline Relative $\mathrm{VO}_{2} \max (\mathrm{ml} / \mathrm{kg} / \mathrm{min})$ & $45.09(6.87)$ & $49.05(5.33)$ & $42.38(6.59)$ & 1.09 & $<0.01 *$ \\
\hline Wingate peak power/kg (W) & $10.29(1.42)$ & $11.07(1.66)$ & $9.76(0.95)$ & 1.02 & $0.01 *$ \\
\hline Wingate mean power/kg (W) & 7.17 (1.19) & $8.08(0.80)$ & $6.55(0.99)$ & 1.67 & $<0.0001^{*}$ \\
\hline Vertical jump (in) & $15.99(3.64)$ & $19.12(2.89)$ & $13.85(2.32)$ & 2.06 & $<0.001^{*}$ \\
\hline Sit and reach $(\mathrm{cm})$ & 18.32 (4.03) & $16.26(3.79)$ & $19.73(3.64)$ & -0.94 & $0.01^{*}$ \\
\hline Bucket carry (m) & $384.94(76.24)$ & 352.77 (83.61) & $409.54(61.72)$ & -0.80 & $0.04 *$ \\
\hline Back squat (reps) & 53.37 (29.16) & $64.38(27.21)$ & $44.94(28.49)$ & 0.69 & 0.07 \\
\hline Deadlift (reps) & $14.47(12.07)$ & $17.92(13.26)$ & $11.82(10.73)$ & 0.52 & 0.18 \\
\hline Arm hang (s) & $55.33(31.55)$ & $67.23(23.63)$ & $46.24(34.39)$ & 0.69 & 0.07 \\
\hline OCR history (years) & $2.78(2.32)$ & $2.75(2.18)$ & $2.79(2.47)$ & -0.02 & 0.96 \\
\hline SGX (max burpees in 5 min) & $68.63(14.59)$ & $72.46(16.21)$ & $65.71(12.96)$ & 0.47 & 0.21 \\
\hline 400-meter run (s) & $118.30(20.04)$ & $105.46(10.80)$ & $128.12(20.11)$ & -1.33 & $0.001^{*}$ \\
\hline 1-mile run (min) & $8.30(1.37)$ & $7.42(0.80)$ & $8.97(1.35)$ & -1.33 & $0.001^{*}$ \\
\hline
\end{tabular}

$\mathrm{SD}$ - standard deviation, $\mathrm{BMI}$ - body mass index, $\mathrm{VO}_{2} \max$ - maximal oxygen uptake, OCR - obstacle course racing,

SGX - Spartan OCR training program

* statistically significant differences between men and women

Table 2. Bivariate associations between demographic, physiological, strength, and performance characteristics and race time

\begin{tabular}{|c|c|c|c|c|c|c|}
\hline \multirow{2}{*}{ Variable } & \multicolumn{2}{|c|}{ Combined $(n=32)$} & \multicolumn{2}{|c|}{ Men $(n=13)$} & \multicolumn{2}{|c|}{ Women $(n=19)$} \\
\hline & Beta $(S E)$ & $p$ value & Beta $(S E)$ & $p$ value & Beta $(S E)$ & $p$ value \\
\hline Age & $0.43(0.18)$ & $0.03 *$ & $0.40(0.18)$ & $0.04 *$ & $0.66(0.26)$ & $0.02 *$ \\
\hline BMI & $0.49(0.78)$ & 0.54 & $0.02(0.86)$ & 0.98 & $1.94(1.12)$ & 0.10 \\
\hline Fat mass & $1.12(0.29)$ & $<0.001^{*}$ & $0.41(0.48)$ & 0.41 & $1.32(0.42)$ & $<0.01^{*}$ \\
\hline Lean mass & $-0.42(0.17)$ & $0.02^{*}$ & $-0.24(0.45)$ & 0.59 & $-0.02(0.61)$ & 0.97 \\
\hline Relative $\mathrm{VO}_{2} \max$ & $-1.15(0.19)$ & $<0.001^{*}$ & $-1.31(0.23)$ & $0.002 *$ & $-1.01(0.31)$ & $<0.01^{*}$ \\
\hline Wingate peak power/kg & $-4.63(1.07)$ & $0.001^{*}$ & $-3.85(0.89)$ & $0.001^{*}$ & $-4.45(2.51)$ & 0.09 \\
\hline Wingate mean power/kg & $-6.47(1.12)$ & $<0.001 *$ & $-5.96(2.44)$ & $0.03 *$ & $-7.33(1.91)$ & $0.01 *$ \\
\hline Vertical jump & $-1.86(0.41)$ & $<0.001^{*}$ & $-1.47(0.72)$ & 0.07 & $-2.63(0.92)$ & $0.01 *$ \\
\hline Sit and reach & $-0.29(0.47)$ & 0.54 & $-0.16(0.64)$ & 0.80 & $-1.50(0.62)$ & $0.03 *$ \\
\hline Bucket carry & $-0.04(0.02)$ & 0.11 & $-0.04(0.03)$ & 0.12 & $-0.11(0.03)$ & $0.01 *$ \\
\hline Back squat & $-0.25(0.04)$ & $<0.001^{*}$ & $-0.21(0.06)$ & $0.01 *$ & $-0.24(0.07)$ & $0.001 *$ \\
\hline Deadlift & $-0.58(0.12)$ & $<0.001^{*}$ & $-0.44(0.13)$ & $0.01 *$ & $-0.62(0.19)$ & $<0.01 *$ \\
\hline Arm hang & $-0.22(0.04)$ & $<0.001^{*}$ & $-0.10(0.10)$ & 0.32 & $-0.23(0.05)$ & $<0.01^{*}$ \\
\hline OCR history & $0.30(0.83)$ & 0.72 & $0.44(1.11)$ & 0.69 & $0.20(1.05)$ & 0.84 \\
\hline SGX & $-0.54(0.08)$ & $<0.001^{*}$ & $-0.37(0.10)$ & $0.01 *$ & $-0.65(0.11)$ & $<0.001^{*}$ \\
\hline 400-meter run (s) & $0.43(0.05)$ & $<0.001^{*}$ & $0.61(0.13)$ & $0.01^{*}$ & $0.42(0.07)$ & $<0.001 *$ \\
\hline 1-mile run (min) & $6.43(0.71)$ & $<0.001^{*}$ & $8.63(1.61)$ & $<0.001 *$ & $6.41(1.07)$ & $<0.001^{*}$ \\
\hline
\end{tabular}

$\mathrm{SE}$ - standard error, BMI - body mass index, $\mathrm{VO}_{2} \mathrm{max}$ - maximal oxygen uptake, OCR - obstacle course racing,

SGX - Spartan OCR training program

* statistically significant associations between that variable and simulated OCR performance 
Table 3. Associations between demographic, physiological, strength, and performance characteristics and race time after statistical adjustment*

\begin{tabular}{lcc}
\hline \multirow{2}{*}{ Variable } & \multicolumn{2}{c}{ Combined $(n=32)$} \\
\cline { 2 - 3 } & \multicolumn{1}{c}{ Beta $(S E)$} & $p$ value \\
\hline BMI & $-0.06(0.42)$ & 0.88 \\
Fat mass & $0.12(0.22)$ & 0.61 \\
Lean mass & $-0.24(0.21)$ & 0.27 \\
Relative $\mathrm{VO}_{2}$ max & $-0.23(0.25)$ & 0.62 \\
Wingate peak power/kg & $-1.59(0.88)$ & 0.08 \\
Wingate mean power/kg & $-0.04(1.77)$ & 0.98 \\
Vertical jump & $-0.74(0.41)$ & 0.08 \\
Sit and reach & $0.12(0.30)$ & 0.68 \\
Bucket carry & $-0.04(0.01)$ & $0.001^{*}$ \\
Back squat & $-0.06(0.05)$ & 0.26 \\
Deadlift & $-0.14(0.11)$ & 0.22 \\
Arm hang & $-0.07(0.04)$ & 0.11 \\
OCR history & $0.35(0.43)$ & 0.43 \\
SGX & $-0.20(0.097)$ & 0.05 \\
400-meter run & $-0.13(0.29)$ & 0.64 \\
1-mile run & $6.33(0.97)$ & $<0.001^{* *}$ \\
\hline
\end{tabular}

SE - standard error, BMI - body mass index,

$\mathrm{VO}_{2} \max$ - maximal oxygen uptake, OCR - obstacle course racing, SGX - Spartan OCR training program

* model adjusted for age, sex, and 1-mile-run time

** statistically significant associations between

that variable and simulated OCR performance

$\mathrm{VO}_{2}$ max explained $81 \%$ of the variance in 10 -mile race time, while the velocity at $\mathrm{VO}_{2} \max$ was the best predictor of race time [12]. OCR presents a unique challenge to participants and researchers since it includes single-mode activities such as running, but also involves obstacles that present other functional challenges that require the ability to produce work at rates above those possible from aerobic metabolism alone. Thus, it is perhaps not surprising that in addition to aerobic fitness, measures of anaerobic energy production and muscle endurance were also associated with OCR performance. Research conducted on performance predictors for HIFT (specifically, CrossFit workout performance) has revealed results similar to those of the present study. For example, Dexheimer et al. [8] examined relationships between typical laboratory performance measures $\left(\mathrm{VO}_{2} \max\right.$, Wingate performance, critical running speed, and muscle strength) and 3 different CrossFit workouts ranging from $<3$ minutes to $<15$ minutes duration. Not surprisingly, $\mathrm{VO}_{2}$ max was the best predictor of the variance for the longest workout, but mean and peak Wingate power and 1-repetition max back squat, strict shoulder press, and deadlift were also associated with performance time. Other studies have reported similar results $[6,7]$.
The purpose of this study was not to explicitly examine differences between sexes in variables related to OCR performance, but this still merits some discussion. The majority of obstacles are the same for men and women during OCR; the only differences tend to be for obstacles that involve weighted objects, with women having lighter loads than men. We found that men were faster, taller, heavier, leaner, had higher $\mathrm{VO}_{2}$ max values when adjusted for body weight, higher values for Wingate peak and mean power adjusted for body weight, and better vertical jump scores. Conversely, women were more flexible and covered greater distances during the bucket carry test. The study by McLaughlin et al. [12], which examined running performance, did not differentiate between men and women, so it is unclear whether the same determinants of performance shown in men apply to women. Similarly, the work of Bellar et al. [6] included all men, while studies by Butcher et al. [7] and Dexheimer et al. [8] involved small numbers of women ( $n=4$ and 5, respectively), who were not analysed separately from the men. Future research, therefore, should incorporate sufficient sample sizes to conduct separate sexspecific analyses.

In addition to physiological characteristics, experience with a given sport or modality of exercise should also intuitively be related to performance outcomes. In the present study, we did not observe relationships between OCR experience and simulated OCR performance. Contrary to this, Bellar et al. [6] reported that CrossFit experience ( $\geq 12$ months) was significantly associated with 2 separate workouts. Similar studies from Dexheimer et al. [8] and Butcher et al. [7] did not examine associations between experience and workout performance. However, in these studies, participants had ca. 4 years' experience compared with the Bellar et al. [6] study, which had a group of 'experienced' and of 'naïve' athletes. The participants in the current study, while presenting similar aerobic fitness values, generally had less experience with OCR compared with the subjects in the aforementioned CrossFit studies, despite a large range in the current study (from 6 months to 7.25 years). Given the heterogeneity in OCR experience and the small sample size, our lack of a relationship between experience and performance is not unexpected.

\section{Limitations}

Our study is not without some limitations. First, all participants came from the same training facility and generally followed the same training program. How- 
ever, this could also be seen as a strength as workouts were hence controlled during testing. Second, we had a large range of abilities and experience in the study sample. Third, we did not quantify peak running speed or lactate threshold with laboratory testing. The running surface for the simulated OCR was different from typical OCR surfaces, and we only examined a short distance OCR. Longer distances would likely yield different results. Finally, our sample size was on the small side, and unequal numbers of men and women precluded us from conducting a more thorough comparison on the basis of sex. The small sample size also prevented us from performing multivariate regression analyses. Regarding future studies, it would be useful to include larger sample sizes and conduct field studies to determine the physiological demands of OCR across different distances and durations.

\section{Conclusions}

The current study examined relationships between laboratory and gym measures of performance and simulated OCR. The results have implications for performance and training of individuals participating in OCR. The first is that aerobic capacity is an important component of OCR performance. The second is that muscle strength and anaerobic performance are also significant factors and come into play with certain obstacles, such as bucket and/or sandbag carries, monkey bars, and rope climbs. Therefore, athletes training for OCR and coaches need to focus on all components of physical fitness in order to achieve optimal success.

\section{Acknowledgments}

We thank the Fall 2018 KINE 326 class for assistance with data collection and MROC Obstacle Course Racing Training Center in Oceanside, USA for their support of this study. No funding was received for this study.

\section{Disclosure statement}

No author has any financial interest or received any financial benefit from this research.

\section{Conflict of interest}

WT is an employee of MROC Obstacle Course Racing Training Center in Oceanside, USA. RFA and MMS state no conflict of interest.

\section{References}

1. Mullins N. Obstacle course challenges: history, popularity, performance demands, effective training, and course design. J Exerc Physiol Online. 2012;15(2):100128.

2. Rawdon CL. Performance variables of obstacle course racing and recommendations for programming in novice and intermediate competitors. Strength Cond J. 2016; 38(5):1-17; doi: 10.1519/SSC.0000000000000253.

3. Spartan. Available from: https:/www.spartan.com/en/.

4. Tough Mudder. Available from: https://toughmudder. $\mathrm{com} /$.

5. Dern N. Why are obstacle-course races so popular? The Atlantic. 2018. Available from: https://www. theatlantic.com/health/archive/2018/07/why-are-obstacle-course-races-so-popular/565130/.

6. Bellar D, Hatchett A, Judge LW, Breaux ME, Marcus L. The relationship of aerobic capacity, anaerobic peak power and experience to performance in CrossFit exercise. Biol Sport. 2015;32(4):315-320; doi: 10.5604/ 20831862.1174771.

7. Butcher SJ, Neyedly TJ, Horvey KJ, Benko CR. Do physiological measures predict selected CrossFit ${ }^{\circledR}$ benchmark performance? Open Access J Sports Med. 2015; 6:241-247; doi: 10.2147/OAJSM.S88265.

8. Dexheimer JD, Schroeder ET, Sawyer BJ, Pettitt RW, Aguinaldo AL, Torrence WA. Physiological performance measures as indicators of CrossFit ${ }^{\circledR}$ performance. Sports. 2019;7(4):93; doi: 10.3390/sports7040093.

9. Baghurst T, Prewitt SL, Tapps T. Physiological demands of extreme obstacle course racing: a case study. Int J Environ Res Public Health. 2019;16(16):2879; doi: 10.3390/ijerph16162879.

10. American College of Sports Medicine. ACSM's guidelines for exercise testing and prescription. Baltimore: Lippincott Williams \& Wilkins; 2013.

11. Cohen J. Statistical power analysis for the behavioral sciences. New York: Lawrence Erlbaum Associates; 1988.

12. McLaughlin JE, Howley ET, Bassett DR Jr, Thompson DL, Fitzhugh EC. Test of the classic model for predicting endurance running performance. Med Sci Sports Exerc. 2010;42(5):991-997; doi: 10.1249/MSS.0b013e3181c 0669d. 